\title{
Sizing, Fractionation and Mixing of Biological Objects Via Microfabricated Devices
}

\author{
Olgica Bakajin, R. Carlson, C.F. Chou, S.S. Chan, \\ C. Gabel, James Knight, Ted Cox and R.H. Austin \\ Departments of Physics and Biology \\ Princeton University, Princeton, NJ 08544
}

\begin{abstract}
SUMMARY
One aspect of micro and nanofabrication that has not been exploited a great deal has been the ability to make structures to probe and separate complex mixtures using designed environments. We will give three brief examples of such second-generation uses of microfabrication, as opposed to simply shrinking the size of the vessels or tubes used in macroscopic lab environments. The three examples chosen are blood cell fractionation and cell sorting, asymmetric brownian motion fractionation and ultra-high speed fluid mixing.
\end{abstract}

\section{INTRODUCTION}

Silicon micromachining has opened up a new world of sub-micron spaces in which you can study biological objects on a scale commensurate with their size and operational environment. Since silicon micromachining is so highly sophisticated in terms of technology, it is possible to design and construct highly creative structures which can probe specific aspects of a biological object. Such structures can also be very practical and useful in applied areas such as biotechnology.

The manipulation and sorting of biological particles poses unique challenges to microfabrication because of the complex physical properties of biological particles. These properties range from size (DNA is an extremely long but thin polymer while the cell is a compact sphere) to adhesive properties (white blood cells are selectively extremely sticky while red blood cells are designed to be quite non-adhesive). An even more important issue is the fact that each biological particle, whether it be the sequence of a DNA fragment or a white blood cell, is unique. Often it is vital to ascertain the uniqueness of the particles, to sort them and find a very rare individual in a population of millions. Many biological sensors rely upon highly specific molecular interactions to sort out a heterogeneous population. We have taken a different approach, the fabrication of micron to nanometer structures which probe the physical properties of biological particles and separate them based on their interactions with physical obstacles.

A related issue to that of structure selection is surface properties. Biological objects, in particular proteins and cells, are notoriously adhesive, and in the microfabricated world surface is everything so the problems of sample loss are only compounded. The silicon dioxide surface of a silicon wafer, or the glass surface of a etched glass wafer, is not a good surface for biology since it can be quite adhesive and in our hands will stop every white blood cell in a drop of blood as it enters a micromachined volume. While one can devise lotions and potions consisting of complex proteins such as bovine serum albumin, the presence of another complex biological component can only serve to further confound the delicate analysis of a already complex object. Thus, we have expended a great deal of work to find very clean and simple surfaces which will not by themselves selective bind biological objects. The materials of choice, silicone elastomers, offer three attractive advantages: they can be used as replicas of negative image micromachined master wafers so that very inexpensive copies of the structures can be made, they selfseal hermetically to clean surfaces so many of the bonding problems in micromachining are removed, and there are ways to selectively modify the surface of silicones if so desired.

\section{DEVICE FABRICATION AND MODIFICATION}

Silicone replicas were made by pouring 5 grams of degassed silicone mixed $1 / 10$ with catalyzing agent over a 3" wafer. The silicone was polymerized by putting the wafer in a $80^{\circ} \mathrm{C}$ oven for 15 minutes. The silicone replica was peeled off the master wafer and placed for 2 minutes in an oxygen plasma vacuum chamber (Herrick Scientific). This is a crucial step which makes the silicone hydrophilic for approximately $1 / 2$ hour. The hydrophilic silicone was placed against the desired window material and immediately wet with water. If the structure is to withstand positive hydrostatic pressures the silicone sealed structure was placed in a water bath at $80{ }^{\circ} \mathrm{C}$ for 24 hours to enhance adhesion top the mating surface. There are further details about this process which we would be glad to discuss.

\section{EXPERIMENTAL RESULTS}

Cell Sorting: Cells respond to their environments in a variety of ways. For the human leukocyte, this complex environment consists of the human circulatory system, the study of which is most often physiological in nature. Here we demonstrate, using physiological flow conditions and a 
microfabricated array of channels with length scales similar to those of human capillaries (see Figure 1), a novel interaction of leukocytes with their physical environment [1]. Using vital chromosome stains and cell specific, fluorochrome labeled antibodies, we observe that the eventual adhesion of the leukocytes to the array does not occur in a manner consistent with a random process (Figure 2). Further, the distribution of cells in the arrays displays a strong dependence on cell type and nuclear morphology, with granulocytes penetrating a smaller distance than lymphocytes and interacting with the lymphocytes in a selfexclusionary manner. The physical distortion of the cells is the same as they experience in vivo, and we propose that this complex non-random behavior is due to a hydrodynamic shear and deformation activated change in the cells relevant to observed in vivo behavior. Because we use reversibly sealed arrays, in the near future we will be able to probe differences among individual stuck cells, either physically using techniques such laser tweezers or atomic

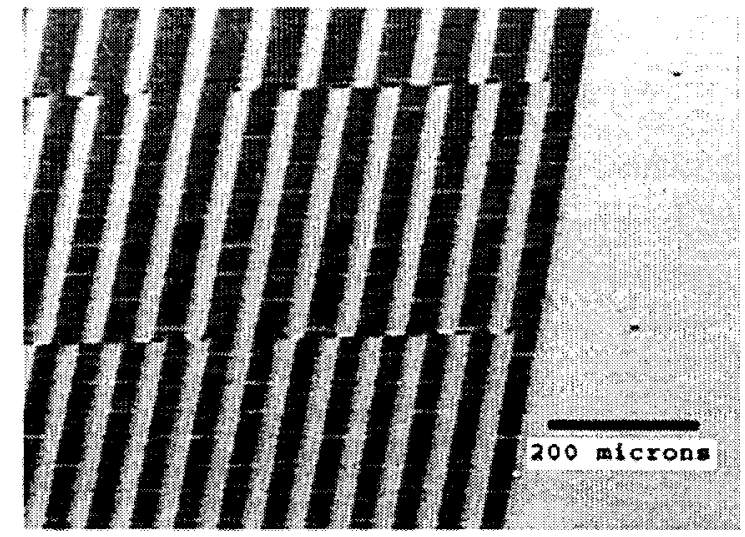

Figure 1. Multiple-length array for cell separation
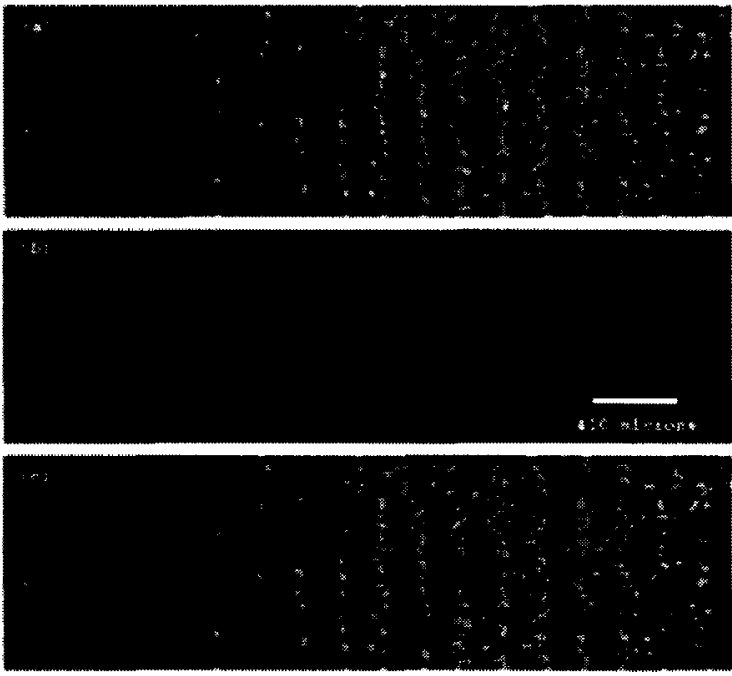

Figure 2. Anti-body fluorescence of labeled leukocytes. Top panel is the raw image, middle is the T-lymphocyte cells, bottom is granulocytes. force microscopy, or via blotting transfer techniques to explore the biochemical variations among cells.

Brownian motion as a fractionating device: In a microfabricated world it is possible to design structures very difficult to realize in normal materials, here we discuss how asymmetry in structures gives rise to a rectified brownian motion. Tom Duke [2] and Deniz Ertas [3] realized independently that by making using a combination of asymmetric structures, flow and brownian motion it would be possible to construct devices which in a continuous injection manner could fractionate objects based upon their diffusion constants. The basic idea is fairly simple: one uses a wall and an opening which brings molecules down along the wall. Because the wall breaks left-right symmetry those molecules with a larger diffusion constant will move further from the wall on the average than molecules of small diffusion constants. If two exits exist at some distance down the wall the smaller molecules will be more likely to exit far from the wall than large molecules. The major advantage here is that the ejection is continuous and so is the extraction, so that you don't have to run as a single event. Ertas proposed a conceptually elegant but difficult to fabricate structure, while Duke and Austin proposed a very simple structure simply consisting of rectangles rotated at an angle to the applied field direction. Figure 3 shows the oriented block configuration of Duke and Austin, while Figure 4 shows how injected molecules would be expected to migrate at differing angles to the applied field direction.

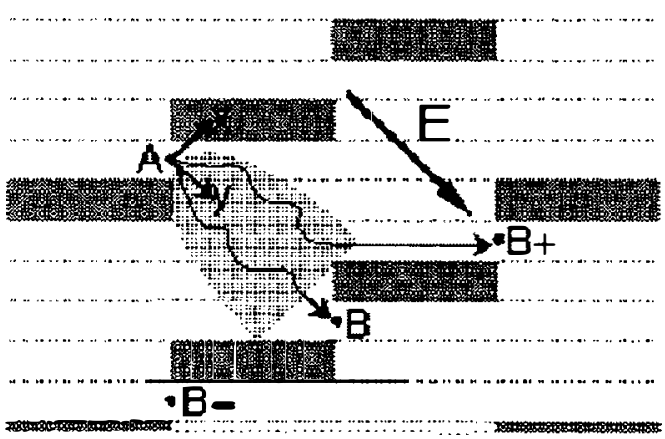

Figure 3. An asymmetric array of blocks. The electric field points $45^{\circ}$ to the lower right. 


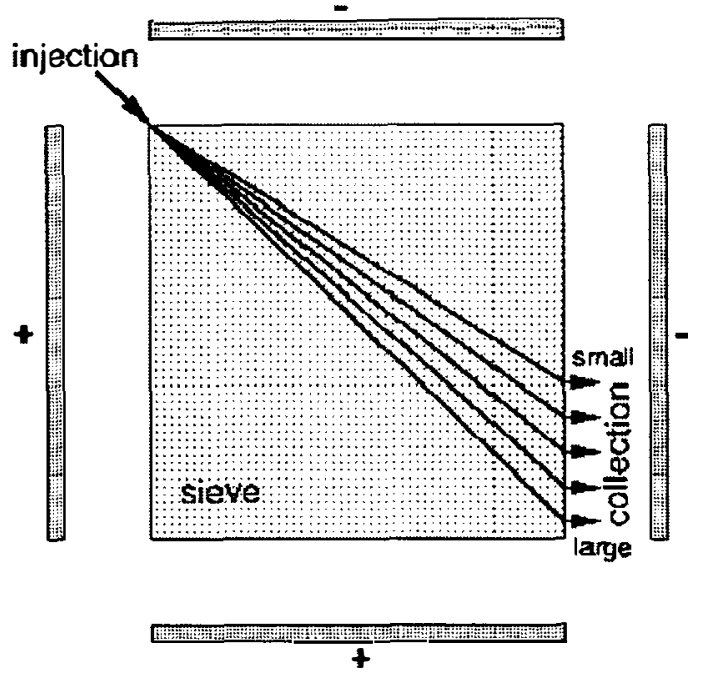

Figure 4. The deflection due to rectified brownian motion.

The performance of such devices will be governed by the dimensionless quantity $\mathrm{D} / \mathrm{va}$, where $\mathrm{D}$ is the diffusion constant of the object, $v$ is its electrophoretic speed and a is the characteristic opening of the asymmetric pores determines resolution.. Intuitively, there is a "sweet spot" for the resolution of this device. If $\mathrm{D}$ is too large or va too small, blurring occurs because of too rapid diffusion. If $D$ is too small or va too large, most objects go straight down through the center channels. Figure 5 shows how the normalized resolution of the channels shown in Figure 3 changes as a function of $\mathrm{D} / \mathrm{va}$.

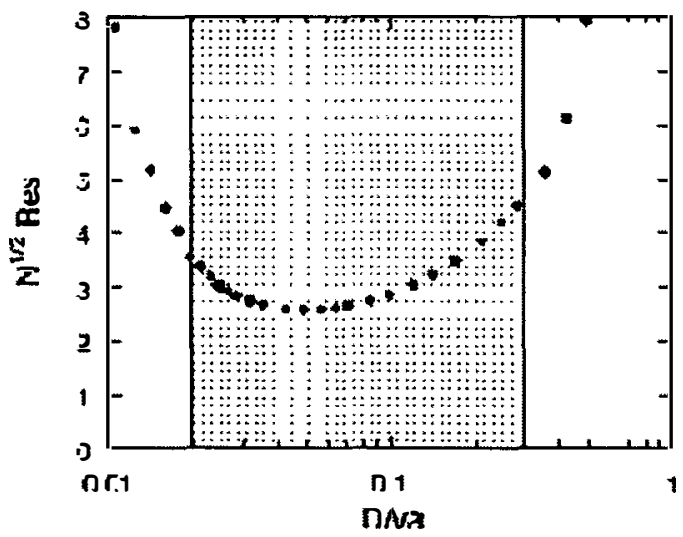

Figure 5. The normalized resolution of the array shown in Figure 3 as a function of D/va

This is very much work in progress. We should be able to show you at the meeting video of the actual fractionation occurring, or explain to you why it doesn't work as well as it should.

Micro/Nano Fluidics for Ultra-rapid Mixing: Mixing Nanoliters in Microseconds Mixing of fluids at the macroscopic scale normally occurs at high Reynolds numbers via turbulence (the cream in your coffee this morning). The Reynolds number $R_{e}$ is a measure of the ratio of (a) the linear momentum/time transported past a fixed point in space carried by moving fluid of density $\rho$ and speed $v$ to (b) the rate at which the momentum is lost due to the shearing viscosity of the fluid $\eta$ as it moves with characteristic shearing length $L$ : $\mathrm{R}_{\mathrm{e}}=\mathrm{a} / \mathrm{b}=\rho \mathrm{vL} / \eta$. In the micron world of microfabrication $\mathrm{R}_{\mathrm{e}}$ is always well below the turbulent threshold of about 1000 even at $1 \mathrm{~m} / \mathrm{s}$ fluid velocities.

Thus, in the microfabricated world mixing cannot occur via turbulence but rather only through brownian diffusion, a process we exploited in the last section to fractionate materials. The classic example involving how long it would take Caesar's flatulence to reach the rear of the Roman Senate us usually cited in freshman physics as an example of how slow diffusion is, since the characteristic $\tau$ to diffuse a distance $x$ is given by: $\tau=x^{2} / 2 D$ where $D$ is the diffusion constant of the object in the solvent. This doesn't necessarily doom mixing to long time scales however. If you make things REALLY small, like nanometers, you can achieve sub-microsecond "mixing" times via diffusion. Further, since the process is diffusive and not turbulent here is no "dead time" in a diffusive mixer but instead a calculable continuous process.

The next question is then: how can you possibly make fluid streams moving at meters/sec $100 \mathrm{~nm}$ wide? Via hydrodynamic focusing, a well understood in the conventional flow cell cytometer. We describe in this report a microfluidic, continuous-flow mixer capable of diffusive mixing times of less than ten microseconds. This is achieved without inducing turbulence and in an open architecture that reduces dead time by permitting observation of the entire mixing process. Figure 5 is an epifluorescence image of the microfabricated device: four rectangular channels, 10 microns deep and wide, intersect at the center. Fluorescent dye labels the flow from the inlet channel and appears bright, while nonfluorescent buffer flows from the side channels. The side flow squeezes, or 'hydrodynamically focuses', the inlet flow into a thin stream that exits the intersection sheathed in buffer fluid. The focusing width can be controlled by varying the relative pressures driving the side and inlet flows, and widths as small as 20 nanometers have been measured.

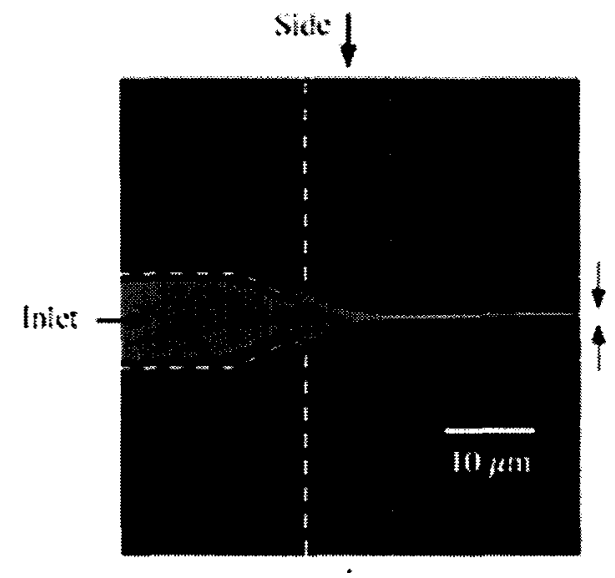

Figure 6. Epifluorescence image of a hydrodynamic jet forming a sub-micron wide jet. Dotted lines show outline. 
At such small length scales, molecules from the side flow rapidly diffuse across the inlet stream, resulting in fast mixing. resolution better than a microsecond per micron. A further consequence of the focusing is the small sample volume consumed by the mixer. The volume flow rates of the focused reactant stream are typically on the scale of nanoliters per second, over three orders of magnitude less than the flows in comparable turbulent mixers (4). While this renders the technique undesirable for mixing coffee, it is ideal for use with expensive biochemical samples.

It is fairly simple under conditions of low $\operatorname{Re}$ to calculate the in/out fluid patterns, since there is a linear relation between the volume of fluid flow $\mathrm{Q}$ in liters/sec and the applied pressure drop $\Delta \mathrm{P}$, in other words, the fluid flow looks like current flow in a resistive circuit. The pressures $\mathrm{P}$ then become voltages $\mathrm{V}$, and the jet that is formed now is the current injected from the center "wire". The critical parameter is the ratio of the side pressure $P_{S}$ to the center pressure $P_{c}$, which we call $\alpha$. Figure 7 shows a comparison of the calculated jet width using the resistive net shown in the insert to the measured jet width as determined from integrated jet intensity. Note that at pinch-off the jet width goes to approximately 20 $\mathrm{nm}$ width! The insert shows how the device can resolve shorter times as $\alpha$ approaches pinch-off.

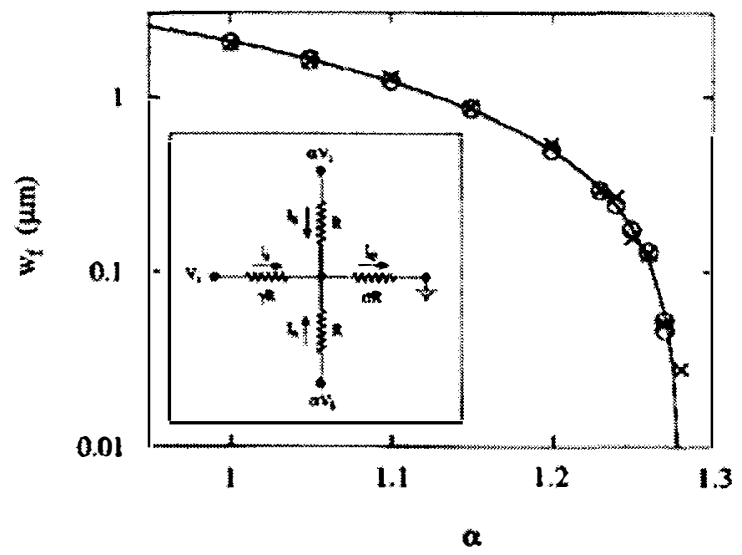

Figure 7. Measured vs. calculated jet width.

A jet width of 20 nanometers moving at $1 \mathrm{~m} / \mathrm{sec}$ gives a time resolution for mixing yielding resolution better than a microsecond per micron. A further consequence of the focusing is the small sample volume consumed by the mixer. The volume flow rates of the focused reactant stream are typically on the scale of nanoliters per second, over three orders of magnitude slower than the rates in comparable turbulent mixers (4). While this device is rather undesirable for mixing coffee, it is ideal for use with expensive biochemical samples.

We have begun experiments utilizing this technology. Figure 8 shows the results of a fluorescence quenching experiment where iodine molecules in the side solution quench via overlap of electronic orbitals the emission of fluorescein molecules. Scanning along the jet with a digital stage on the microscope allows one to measure in a steady state way ultra-rapid mixing kinetics, in this case with a resolution of less than 10 microseconds.

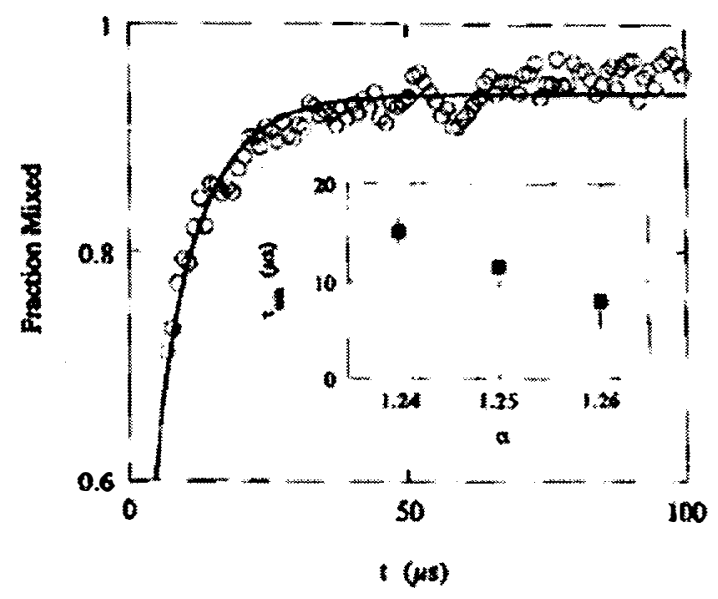

Figure 8. Quenching kinetics of fluorescein.

Now that this technology is well understood [5], we are proceeding with protein folding experiments both using fluorescence quenching, and through etched 400 micron deep channels time-resolved X-ray scattering at CHESS, the Cornell synchrotron light source in collaboration with Sol Gruner and Lois Pollack.

\section{CONCLUSIONS}

There are many exciting possibilities for microfabricated structures in biology that go beyond making the same things smaller. Our devices are actually quite simple to make, but we do hope show some of the potentials of the technology.

\section{ACKNOWLEDGMENTS}

The authors gratefully acknowledge financial support from ONR, NIH, Perkin Elmer, Hewlett Packard and Caliper Technologies.

\section{REFERENCES}

[1] R. H. Carlson, C. V. Gabel, S. Chan and R Austin, " Self-Sorting of White Blood Cells in a Lattice", Phys. Rev. Lett. 15 (1997) 2149-2152.

[2] T. A. J. Duke and R. H. Austin, "Microfabricated Sieve for the Continuous Sorting of Macromolecules", Phys. Rev. Lett. 16 (1998) 1552-1555.

[3] D. Ertas, "Lateral Separation of Macromolecules and Polyelectrolytes in Microlithographic Arrays", Phys. Rev. Lett. 16 (1998) 1548-1551

[4] H. Strehlow, Rapid Reactions in Solution (VCH Publishers, New York, 1992)

[5] J. Knight et al. In Press, Phys. Rev. Lett. 